\title{
Safer cycling in the urban road environment: study approach and protocols guiding an Australian study
}

\author{
Mark Stevenson, ${ }^{1}$ Marilyn Johnson, ${ }^{2}$ Jennie Oxley, ${ }^{1}$ Lynn Meuleners, ${ }^{3}$ Belinda Gabbe, ${ }^{4}$ \\ Geoffrey Rose ${ }^{2}$
}

${ }^{1}$ Monash University Accident Research Centre, Monash Injury Research Institute, Monash University, Melbourne, Australia

${ }^{2}$ Faculty of Engineering, Institute of Transport Studies, Monash University, Melbourne, Australia

${ }^{3}$ Curtin-Monash Accident Research Centre, Curtin University, Perth, Australia ${ }^{4}$ School of Public Health and Preventive Medicine, Monash University, Melbourne, Australia

\section{Correspondence to} Professor Mark Stevenson, Monash University Accident Research Centre, Building 70, Clayton Campus, Monash University, Melbourne, VIC 3800, Australia;

Mark.stevenson@monash.edu

Received 30 April 2014 Revised 11 May 2014

Accepted 21 May 2014 Published Online First 10 June 2014

\section{ABSTRACT}

Background Much of the research into cyclist safety in Australia has focused on behaviour with less focus on the impact of the urban transport environment on cyclist safety. A greater understanding of the urban transport system and the improvements needed to create a safer cycling environment are essential if cyclists are to be safe and increased cycling participation targets are to be achieved. The proposed study will use existing cyclist crash data along with unique cyclist exposure data to develop road infrastructure prototypes that improve cyclists' safety and evaluate the effectiveness of these prototypes in a cycling simulator.

Methods and design This study will be conducted in two Australian cities namely Perth and Melbourne as both cities have policies that strongly advocate cycling. Two methods of data collection will be employed: (1) in-depth crash investigations of injured cyclists; and (2) video footage of cyclist exposure through a naturalistic cycling study of non-injured cyclists. The findings from these two methods will be used to develop new urban road design prototypes which will be tested with a sample of cyclists and motorists in safe environment namely, a cycling simulator and a driving simulator.

Discussion By designing and evaluating safer environments for cyclists, this study will identify solutions that reduce the risk of road trauma and importantly, support this alternative mode of transport and thereby contribute to a reduction in traffic-related emissions and pollution and enhance sustainable economic and social connectivity.

\section{BACKGROUND}

The importance of cycling to achieve sustainable transport and national health goals is well recognised by governments nationally and internationally. ${ }^{1-3}$ Bicycles are an environmentally sustainable transport mode that, when substituted for a car, can reduce vehicle congestion, exhaust pollution, improve travel times and improve health. ${ }^{4}$ People who regularly cycle are less likely to be overweight, less likely to suffer from obesity-related diseases including heart disease, diabetes and stroke and have improved mental health. ${ }^{5}$ Cycling popularity is increasing in Australia with cycling participation at the highest rate in over a decade. ${ }^{6}{ }^{7}$ In 2011, an estimated 4 million Australians (18\%) rode a bicycle in the previous week and 8.5 million $(39.6 \%)$ had ridden a bicycle in the previous year. ${ }^{6}$

Despite the many benefits of cycling, cyclists are physically vulnerable road users, especially when they share the road with motorised vehicles. Concurrent with the increase in cycling participation in Australia is an increase in cyclist serious injury crashes. ${ }^{8} 9$ The majority of cyclist crashes occur in the urban road environment ${ }^{10}$ and crashes involving motor vehicles lead to the most serious outcomes for cyclists. ${ }^{11-13}$ The risk of a death for cyclists is 4.5 times greater than car occupants $^{14}$ and a cyclist's risk of serious injury is 3.6 times greater in a collision with a vehicle compared with all other non-vehicle cyclist crash types. ${ }^{15}$ In Australia, a motor vehicle is involved in the majority of cyclist deaths $(86 \%)^{16}$ and serious injury $(75 \%)^{17}$ crashes. These crashes cost the Australian community an estimated \$A1 billion each year (calculated using 2006 values). ${ }^{18}$

The majority of all cyclist crashes occur in urban environments and of those crashes, a significant proportion occur at intersections. To date, there has been substantial research into how the urban environment can be enhanced to improve cyclist safety in the European context which has resulted in significant improvements to road design and infrastructure. Road networks that are inclusive of cyclists, including cycling infrastructure which physically separates cyclists from motorised vehicles, have been considered critical to the success of cycling participation and cyclist safety in many European countries. ${ }^{19-21}$ While very little has been implemented to improve cycling participation and cyclist safety in the USA, one State, Portland, has championed the adoption of safe cycling environments with the retrofitting of cycling-inclusive infrastructure. ${ }^{22} 23$

Much of the research into cyclist safety in Australia has focused on behaviour, ${ }^{24-26}$ with less focus on the impact of the urban transport environment on cyclist safety. Land use planning in Australia has more in common with the USA than many European countries. This is an important point to consider when adapting international examples to the Australian road context. Current attempts of infrastructure to improve cycling safety have had mixed success. Painted bike boxes at intersections are not observed by drivers ${ }^{27}$ and bike lanes are often positioned alongside parallel parking bays that expose cyclists to the risk of opening vehicle doors. ${ }^{28} \mathrm{~A}$ greater understanding of the Australian transport system and the improvements needed to create a safer cycling environment are essential if cyclists are to be safe and cycling participation targets set by the current federal government policies to double cycling participation by 2016 are to be achieved safely. ${ }^{1}$

The aim of the study therefore, is to propose modifications to the urban road environment that will reduce cyclist crashes. The specific objectives 
of the research are (1) to describe the contributing factors to cyclist crashes in urban road environments, (2) identify features of the urban road environment that increase the risk of a cyclist crash, (3) develop road infrastructure prototypes that improves cyclists' safety and (iv) evaluate the effectiveness of the prototypes in a bicycle simulator.

A unique feature of this study is its multidisciplinary approach to investigating cyclist safety. The study will use a combination of in-depth cyclist crash investigations and innovative naturalistic cycling methods to generate unique data sets of crash-involved and non-crash-involved cyclist experiences. Comparisons of the two cycling groups (crash-involved and non-crash-involved) will extend our knowledge of the factors that contribute to cyclist crashes and injury risk and guide the development, testing and implementation of effective road design features and cyclist facilities. This approach represents a fundamental shift in the approach to cyclist safety. By matching cyclist behaviour and crash experience with the actual road environment, the outcomes will have substantial implications for the long-term individual and societal costs of cyclist injuries.

Retrofitting roads to incorporate space for cyclists is expensive and it is important that any investment provides the greatest safety and mobility outcomes to all road users. Findings from the analysis of existing cyclist crash data and the proposed naturalistic cycling study will inform prototypes of new road designs. These prototypes will be evaluated in a cycling simulator and a driving simulator. A cycling simulator will be built for this project that will provide a safe laboratory environment to trial and evaluate prototypes of road designs suitable for safe cycling. This testing will help to ensure any changes to the road space will achieve actual cyclist safety improvements and maximise the benefits of the financial investment in retrofitting roads.

\section{Cyclist safety and public health}

The recent increase of cycling participation in Australia has provided many benefits, but how to safely accommodate cyclists and drivers across the road network remains a significant challenge. The proposed study is fundamental to supporting the Australian Government's aim of doubling the cycling participation rate between 2011 and $2016^{1}$ by addressing infrastructurerelated safety concerns and will provide evidence for the next advances in cyclist safety.

The introduction of evidence-based safer infrastructure and road design, including intersection design and operation, separation of cyclists and vehicles through provision of wellconnected cycle networks, and speed reduction measures in environments where separation is not practical, can provide the quickest and most effective measure to reduce cyclist trauma. Importantly, the study will have national environmental/societal benefits. Australia faces significant environmental challenges and car travel plays a major role in these challenges. By identifying and proposing safer environments for cyclists, this project will support this alternative mode of transport and contribute to a reduction in traffic-related emissions and pollution and enhance sustainable economic and social connectivity.

The study will make a major contribution to strengthen Australia's preventive healthcare by promoting safer cycling participation that will improve key elements of population health. A safer cycling environment has been found to increase cycling participation $^{22}$ and there are substantial financial benefits to be gained. Currently inactivity in Australia costs $\$ 13.8$ billion. An increase in everyday cycling for commuting and local trips can achieve the recommended activity levels and reduce this cost. ${ }^{29}$ Compared with non-cyclists, regular cyclists take one less sick day per year, this equates to $\$ 61.9$ million saved by businesses per year. ${ }^{29}$ In addition to health-related cost savings, the Australian government forecasts that $\$ 13$ billion congestion-related costs would be saved if bicycle use were to double by $2020 .^{30}$

\section{METHODS AND DESIGN \\ Research overview}

This study will be conducted in four stages in two Australian metropolitan areas: Melbourne (total population of 4.1 million) and Perth (total population 2.2 million). These cities have been selected as both have recently adopted policies that strongly advocate cycling as a viable and sustainable transport mode and urgently require research to provide innovations in enhanced road design and operation. Two methods of data collection will be employed: (1) in-depth crash investigations of injured cyclists (Stage 1); and (2) video footage of cyclist exposure through a naturalistic cycling study of non-injured cyclists (Stage 2). The findings from these two methods will be used to develop new urban road design prototypes (Stage 3) unique to the States of Victoria and Western Australia which will be tested in the cycling simulator and driving (Stage 4). Figure 1 provides a schematic representation of the four stages of the study and associated aims.

\section{In-depth cyclist crash study (crash-involved cyclists)}

This stage involves the compilation of data from three prospective studies (each of 12 months duration) involving cyclist crashes in which the cyclist presented to hospital. This is an efficient design as it uses data from one recently completed (November 2011) cycling-related trauma study in which the investigators were involved. The Melbourne Alfred Cyclist Crash Study recruited 145 adult cyclists who presented to the Sandringham and Alfred hospitals (Melbourne metropolitan area). ${ }^{31}$ A second prospective study of patients with cycling-related trauma, involving the current investigators, and which is based at The Alfred and the Royal Melbourne Hospitals (referred to as the Melbourne-Alfred Study) has just been completed (January 2014). In the second study, a further 150 injured cyclists (from the Melbourne metropolitan area) were recruited using the same protocols as the Melbourne-Sandringham Study. The third prospective study, (recruitment to begin in June 2014 for 12 months), will involve 100 patients with cycling-related trauma admitted to Royal Perth Hospital, Sir Charles Gardiner Hospital, Joondalup and Fremantle Hospitals (referred to as the Perth Study).

In the three studies, a standard study protocol (including study interview schedules) has been (or will be in relation to the Perth-Study) applied. All injured cyclists in Victoria were identified after a daily review of admission records (and or the trauma registry) to the respective study hospitals. A research nurse then conducts a face-to-face interview with the injured cyclist during the cyclist's hospital stay. Where patients are discharged prior to contact with the study investigators, telephone contact is made to obtain patient consent and to complete the interview. Importantly, all protocols for the collection of injury data have been based on crash investigation protocols used in the Monash University Accident Research Centre's Australian National Crash In-Depth Study that has been continuously collecting real-world in-depth serious injury crash data since $2000{ }^{11}$

The interview obtains demographic information, details on cycling exposure, a description of the crash, events leading up to the crash and postcrash information. Injury data is obtained from a review of the medical record and/or the trauma registry (at the Alfred Hospital) which includes injury type, injury severity (using the Abbreviate Injury Scale), Glasgow Coma Score, 
Figure 1 Schematic representation of our aims and research stages.

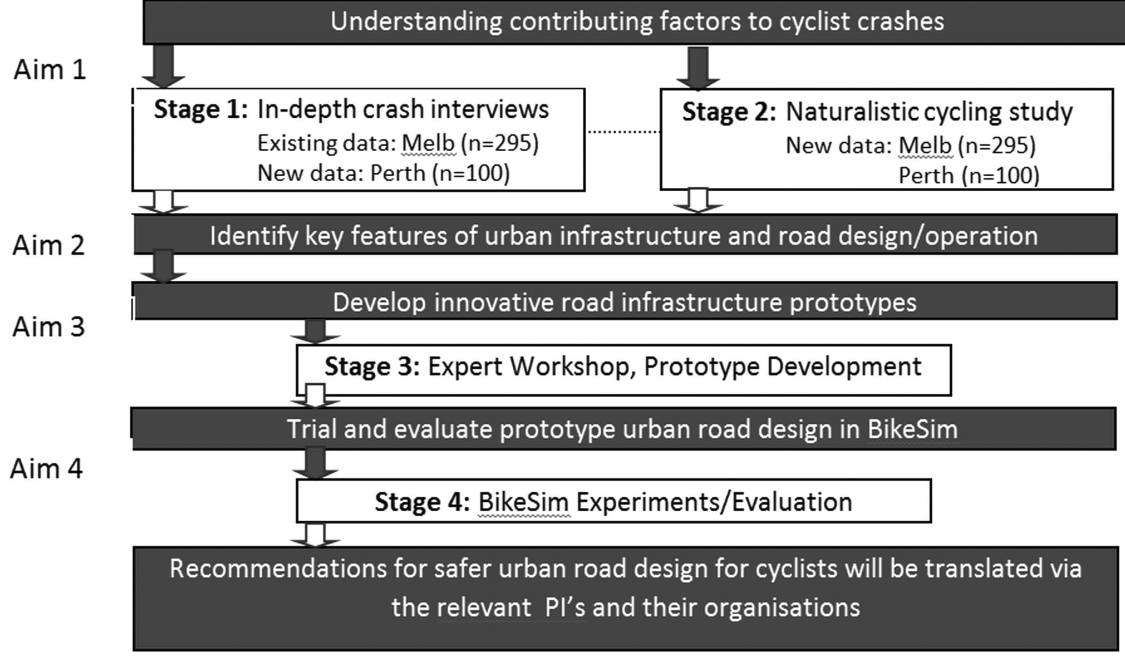

operation report, CT scan report and nursing notes-if available. Salient environmental features including volume, speed and composition of the traffic at the sites, the road type, posted speed limit, number of lanes, footpaths and verges, traffic control, visual obstacles, adjacent land use, associated activity levels and patterns and the presence of on-street parking.

The protocols currently in place in Melbourne have been approved by the hospital ethics committees and the Monash University Human Research Ethics Committee, and Curtin University (Perth) Human Research Ethics approval has also been obtained.

\section{Naturalistic cycling study (non-crash involved cyclists)}

Naturalistic data provides an objective record of the cyclist's point of view when riding on the road and such data can be analysed in a number of ways including: (1) as a representation of a cyclist's exposure and/or (2) to identify factors which contribute to crashes. A number of the investigators have extensive experience in conducting naturalistic cycling studies particularly in relation to identifying factors that contribute to cyclist-driver collision and near-collision events. ${ }^{24} 3233$ In the proposed study, it will be the first time naturalistic methods will be used to investigate the role of urban road design in cyclist crashes by comparing the urban form collected at the crash site with that obtained from non-crash involved cyclists (via exposure data selected using video footage-naturalistic data). Non-injured cyclists will be recruited near on-road crash locations identified from the in-depth cyclist crash studies.

Cyclists will be intercepted as they stop at intersections and trained researchers will identify themselves, describe the purpose of the study and seek the cyclist's involvement and contact details (eg, mobile phone number or email address). All cyclists who provide contact details will be followed up within $24 \mathrm{~h}$ of the roadside intercept.

Each intercepted cyclist who agrees to participate in the study must not have been injured in a cycling crash that required hospitalisation in the 36 months prior to recruitment. This requirement ensures that participants in the naturalistic study have not been involved in a cycling crash resulting in hospitalisation during the recruitment period for participants in the in-depth cyclist crash studies. Participants must be willing to have their bicycle fitted with two cameras to record their cycling trips over a 7-day period (see figure 2). One camera will be mounted on the handlebars (forward view) and the second will be mounted under the seat (rear view). The cameras includes a Global Positioning System (GPS) data logger that records the movement of the cyclists (eg, latitude, longitude, speed, acceleration, braking) across all trips. The camera includes a lithium ion rechargeable battery that allows cyclists to record approximately $2 \mathrm{~h}$ of video and GPS data without interruption.

Video footage and GPS data of each cyclist's trips over a 7-day period will be analysed. The cyclists' exposure data will be stratified by road type travelled on (eg, arterial, subarterial, collector and local roads) and one randomly selected road site (defined as $45 \mathrm{~m}$ of road) will be frequency matched based on the proportion of crashes (from the in-depth crash studies) occurring on the various road types. Once the road site is identified from the video footage, the following data will be obtained either from a visit to the road site or from records obtained from the research study partner organisations namely, volume, speed and composition of the traffic at the sites, posted speed limit, number of lanes, footpaths and verges, traffic control, visual obstacles, adjacent land use, associated activity levels and patterns and the presence of on-street parking.

Figure 2 Cameras fitted to the front and rear of the bicyle.

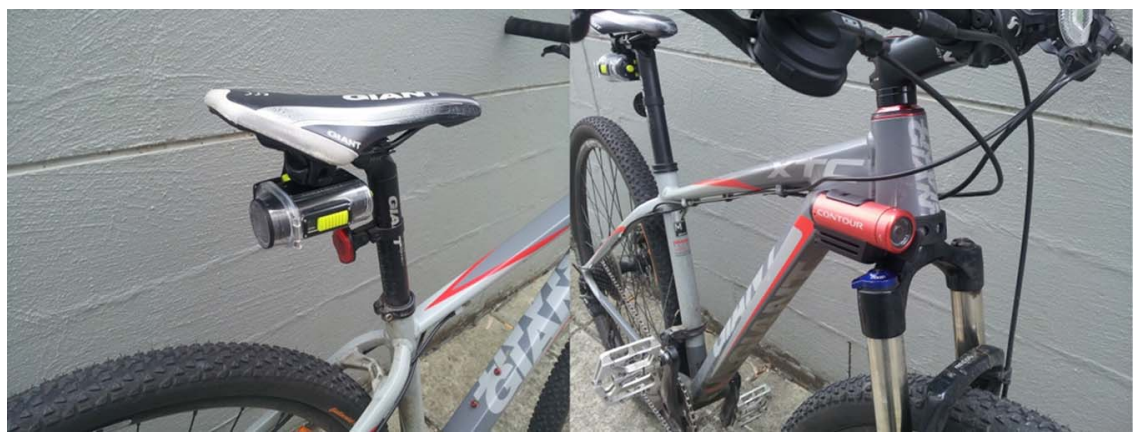




\section{Sample size, data management and analysis}

In total, 295 injured cyclists have been recruited from the Melbourne Alfred Cyclist Crash Study and the Melbourne-Alfred Study. Using the same protocols for the two Melbourne studies, 100 injured cyclists from Perth (Perth Study) will be recruited. A total of 395 non-injured cyclists (295 from Melbourne and 100 from Perth) will have their bicycle instrumented with two video cameras. As described above, randomly selected road sites will be identified from the footage filmed in the naturalistic study. Data on a total of 295 road sites in Melbourne and 100 sites in Perth will be used for modelling variations in road sites (urban form) between sites in which a crash did and did not occur. The proposed sample size will ensure we have sufficient power $(0.8)$ to detect differences (proportional differences $=0.15$ ) in the urban environment between the sites in which crashes did and did not occur. Standard conditional multivariate logistic regression will be used in the data analysis, taking into account the partial matching (eg, road types) of the sites where crashes did and did not occur. ${ }^{34}$

\section{Prototype development of road designs for safe cycling}

Taking the findings from the earlier studies a Kinetic Energy Management Model (KEMM) will be applied when designing enhanced urban prototypes. The $\mathrm{KEMM}^{35}$ provides a means of systematically dissecting the various elements, and stages, of a cyclist's crash: from the exposure of a cyclist to a crash; to the risk of a cyclist being in a crash given exposure; to the potential kinetic energy a cyclist would be exposed to and the resultant levels of kinetic energy transferred to the cyclist, and hence crash injury consequences. As alluded to above, the crash and naturalistic data will provide the basis for the KEMM analysis and the findings will provide a system-wide compilation of factors contributing to unacceptable levels of cyclist risk.

Injury outcomes following a collision between a cyclist and motor vehicle are essentially dictated by the laws of physics and speed, mass differentials, vehicle trajectories, cyclist and cycle separation and impact angles. To develop urban road designs aligned with fundamental safety principles and human injury thresholds, we will develop a Safe System framework for cyclists through which the risk factors for cyclist injury can be ameliorated. A Safe System framework, ${ }^{36} 37$ treats the road system as a whole, identifying the specific needs of various road users, and physically creating conditions that minimise fatal and serious injury outcomes. The framework combines the theories of physics and the knowledge identified in the earlier research stages particularly the cause of the crash stemming from human limitations and existing design deficiencies which will enable us to recognise the thresholds that cannot be violated if a Safe System is to be maintained. Consultation with designers, engineers and representatives of cycling groups will be undertaken in order to develop the cycling-related Safe System framework.

Urban/road designs developed upon the principles identified above are likely to produce a robust environment minimising the risks for cyclists. A template for safer road designs will be used to start discussions in a workshop setting. The template is based on methods successfully applied in developing safe intersection design. ${ }^{38}$ Workshops will be undertaken with relevant stakeholders in cyclist safety and road management, road design experts, urban design and creative design and partner organisations, to identify potential design ideas given the findings highlighted from the preceding research stages.

The resultant designs will be shortlisted based on the criteria defined by the workshop participants. Criteria could include aspects such as, level of safety likely to be produced as a result of the design, objective and subjective safety, compatibility with existing road designs, ease and cost of implementation, impact on the environment and traffic capacity/operations. It is anticipated that designs could include elements such as improved delineation to define bicycle and vehicular travel paths, audibletactile devices, pavement markings, spatial separation of traffic streams (using line marking or low profile physical barriers) and speed management. The selected designs will be produced as functional layout plans. Through further stakeholder consultation and round-table discussions, a select number of designs deemed to have the greatest potential, will be taken to the final stage. A selected design will be developed and then brought to trial using two simulators (cycling and driving).

\section{Simulation evaluation (cycling and driving simulation)}

Simulation techniques are an excellent and established method to test system-based counter measures and will be used to test driver and cyclist performance with different prototypes (road designs). The simulations of the prototypes will be tested twice: once in a cycling simulator that will be purpose-built as part of this study and a second time in the Monash University Accident Research Centre's Advanced Driving Simulator. These simulation techniques will provide the platform to assess selected performance measures including (but not limited to):

1. Bicycle/vehicle-based measures including mean and SD of lateral position, mean speed and speed profiles on approach to critical events and reaction time.

2. Cyclist/driver eye movements including object detection times and fixation durations, assessed using the FaceLab system, providing for a more theoretical hypothesis testing related to attention strategies adopted by road users.

3. Subjective data collected via postride/postdrive interviews regarding cyclist/driver perceptions of the road designs trialled and understanding of required behaviours.

Five simulator studies will be conducted with each study comprising 25 participants from the States of Victoria and Western Australia. This sample size has been found to be sufficient to detect changes resulting from road infrastructure-based manipulations in the simulator. ${ }^{39}$ Between-subject designs will be used predominantly as they provide a more representative behavioural response in cases involving rare events, as the merits of exposing participants to a large number of rare events within a short time period is questionable. ${ }^{39}$ The locations of crashes as identified in Stage 1 will be used to identify the types of locations (intersections/midblocks) where the crash occurred and the circumstances of the crash.

\section{DISCUSSION}

Recent studies have shown the overwhelming health benefits from cycling. For example, a 6-year study that followed 67000 Chinese women in Shanghai found that women who walked and cycled had significantly lower rates of mortality, from all causes, than those who did not. Similarly, in Denmark, a country which has prioritised cycling, cycling to work is associated with a $40 \%$ decrease in mortality among working-age men. ${ }^{40}{ }^{41}$ These are overwhelming findings and yet, these findings cannot be directly translated to Australia (or many other highly motorised countries) as the provision of a safe cycling infrastructure has not been prioritised. Interestingly, for many years China has provided designated cycling lanes for cyclists. These, however, are frequently being removed as a consequence of rapid motorisation. ${ }^{41}$ 
As alluded to earlier, research investigating cyclist safety in Australia has focused on behaviour, ${ }^{24-26}$ with limited focus on the impact of the urban transport environment on cyclist safety. Land use planning in Australia has more in common with the USA than many European countries. This is an important point to consider when adapting international examples to the Australian road context. Consequently, the proposed study will provide a greater understanding of the Australian transport system and the improvements needed to create a safer cycling environment. This research is essential if cyclists are to be safe and cycling participation targets set by the current federal government policies are to be achieved. ${ }^{1}$

Australia, along with other highly motorised countries, faces significant environmental challenges and high levels of car travel plays a major role in these challenges. By identifying and proposing safer environments for cyclists, this study will identify solutions that support this alternative mode of transport and contribute to a reduction in traffic-related emissions and pollution and enhance sustainable economic and social connectivity.

\section{Practical application}

Knowledge translation is a major component of this research. Representatives from partner advocacy groups for cyclists sit on the studies management committee and will contribute to the communication of the research findings to the cycling community and the public at large. We are also committed to enhancing the interface between research and policy, and promoting evidence-informed policy. This will be undertaken by establishing a Translation Task Group (TTG) that will facilitate interaction and debate between the stakeholders and the researchers.

The TTG will facilitate interaction in relation to the research findings. The group will comprise members from the study management group along with key government policy makers responsible for transport planning and road network responsibilities. The TTG representatives will be integral to the findings from the simulation and will be the facilitators for the integration of the research findings into real infrastructure projects.

Contributors MS, MJ, JO, LM, BG and GR contributed to the concept and design of the protocol. All authors reviewed and approved the final version.

Competing interests This work is supported by Australian Research Council Grant Number: LP130100380. MS and BG are supported by National Health and Medical Research Council Fellowships.

Patient consent Obtained.

Ethics approval Australian Research Council, Monash University Human Research Ethics Committee.

Provenance and peer review Not commissioned; externally peer reviewed.

\section{REFERENCES}

1 Austroads. National Cycling Strategy 2011-2016: gearing up for active and sustainable communities. 2010.

2 Department of Transport. Victorian Cycling Strategy, V. Victorian Government, Editor. 2009

3 Department of Transport Western Australia. Bicycle strategy for the 21st Century, P. Western Australian Government, Editor. 2009.

4 Garrard J. Active transport: adults, An overview of recent evidence. 2009. [cited 25 June 2010]. http://www.goforyourlife.vic.gov.au/hav/articles.nsf/practitioners/active_ transport?Open

5 Oja P, Titze S, Bauman A, et al. Health benefits of cycling: a systematic review. Scand J Med Sci Sports 2011;21:496-509.

6 Australian Bicycle Council and Austroads, Australian Cycling Participation. 2011.

7 Department of Communications Information Technology and the Arts. Participation in Exercise, Recreation and Sport Annual Report 2010. 2011

8 Henley G, Harrison JE. Serious injury due to land transport accidents, Australia, 2000-01 to 2007-08, AlHW Injury Research and Statistics Series No 56, Editor. 2011, Australian Institute of Health and Welfare, Australian Government.
9 Sikic M, Mikocka-Walus A, Gabbe B, et al. Bicycle injuries and mortality in Victoria, 2001-2006. Med J Aust 2009;190:353-6.

10 Watson L, Cameron M. Bicycle and motor vehicle crash characteristics. Research Report 251. Melbourne: Monash University Accident Research Centre, 2006.

11 Bostrom L, Nilsson B. A review of serious injuries and deaths from bicycle accidents in Sweden from 1987 to 1994. J Trauma Inj Infect Crit Care 2001;50:900-7.

12 Haileyesus T, Annest JL, Dellinger AM. Cyclists injured while sharing the road with motor vehicles. Inj Prev 2007:13:202-6.

13 Chong S, Poulos R, Olivier, et al. Relative injury severity among vulnerable non-motorised road users: comparative analysis of injury arising from bicycle-motor vehicle and bicycle-pedestrian collisions. Accid Anal Prev 2010;42:290-6.

14 Garrard J, Greaves S, Ellison A. Cycling injuries in Australia: road safety's blind spot? J Aust Coll Road Saf 2010;21:37-43.

15 Rivara FP, Thompson DC, Thompson RS. Epidemiology of bicycle injuries and risk factors for serious injury. Inj Prev 1997;3:110-14.

16 Australian Transport Safety Bureau. Deaths of cyclists due to road crashes. Australian Government, 2006.

17 Henley G, Harrison GE. Serious injury due to land transport accidents, Australia, 2006-07, in Flinders University, AlHW Injury Research and Statistics Series No 53, Editor. Australian Institute of Health and Welfare, Australian Government, 2009.

18 Bureau of Infrastructure Transport and Regional Economics. Road crash costs in Australia. Bureau of Infrastructure Transport and Regional Economics. Canberra, 2006.

19 Pucher J, Buehler R. Making cycling irresistible: lessons from the Netherlands, Denmark, and Germany. Transport Rev 2008;28;4:495-528.

20 Pucher J, Buehler R. Cycling for everyone: lessons from Europe. Transportation Research Board, 2008:58-65.

21 Pucher J, Dijkstra L. Promoting safe walking and cycling to improve public health: lessons from the Netherlands and Germany. Am J Public Health 2003;93:1509-16.

22 Dill J, Carr T. Bicycle commuting and facilities in major U.S. cities: if you build them, commuters will use them. Transp Res Rec 2003;1828:116-23.

23 Pucher J, Dill J, Handy S. Infrastructure, programs, and policies to increase bicycling: An international review. Prev Med 2010;50(Suppl 1):S106-25.

24 Johnson M, Charlton J, Oxley J. Naturalistic cycling study: identifying risk factors for on-road commuter cyclists. Ann Adv Automot Med 2010;54:275-83.

25 De Rome L, Boufous S, Senserrick T, et al. The pedal study: factors associated with bicycle crashes and injury severity in the ACT. The George Institute for Global Health, The University of Sydney, 2011.

26 Schramm A, Rakotonirainy A, Haworth N. How much does disregard of road rules contribute to bicycle-vehicle collisions? National Conference of the Australasian College of Road Safety and Travelsafe Committee of the Queensland Parliament, Brisbane, 2008.

27 Johnson M, Charlton J, Newstead S, et al. Painting a designated space: cyclist and driver compliance at cycling infrastructure at intersections. J Australas Coll Road Saf 2010;21:67-72.

28 Johnson M, Newstead S, Oxley J, et al. Cyclists and open vehicle doors: crash characteristics and risk factors. Saf Sci 2013;59:135-40.

29 Australian Bicycle Council and Cycling Promotion Fund. Australian Cycling, an economic overview. 2011. [cited 12 October 2012]. http://www.austroads.com.au/ abc/images/pdf/the australian_cycling_economy.pdf

30 Department of Infrastructure and Transport. Walking, Riding and Access to Public Transport, Draft report for Discussion, October 2012, A. Government, Editor. 2012: Canberra.

31 Biegler P, Newstead S, Johnson M, et al. MACCS Monash Alfred Cyclist Crash Study. Monash University Accident Research Centre Report Series. No. 311. 2012. Clayton.

32 Johnson M. Cyclist safety: an investigation of how cyclists and drivers interact on the roads, in Monash Injury Research Institute, Monash University Accident Research Centre, Monash University: Clayton, 2011.

33 Johnson M, Charlton J, Oxley J. The application of a naturalistic driving method to investigate on-road cyclist behaviour. J Road Transport Res 2010;19:33-42.

34 Greenland S. Applications of stratified anlaysis methods. In: Modern epidemiology. Rothman J, Greenland S, eds. Philadelphia: Lippincott-Raven, 1998:286-7.

35 Corben B, Camerson M, Senserrick T, et al. Development of the Visionary Research Model_-application to the car/pedestrian conflict. Monash University Accident Research Centre. Report No. 229. Clayton: Monash University, 2004

36 National Road Safety Council. Road Safety Fact Sheet NRSC2, Safe System Approach. 2010.

37 Howard E. Vision Zero and the 'Safe Systems Approach': an International Perspective-OECD/ITF Towards Zero Report, 2008.

38 Corben B, Candappa N, van Nes N, et al. Intersection Safety: meeting Victoria's intersection challenge, Stage 2, Task 5: Generation and Evaluation of Intersection Design within the Safe System Context, 2010.

39 Lenné MG, Rudin-Brown CM, Navarro J, et al. Driver behaviour at rail level crossings: responses to flashing lights, traffic signals and stop signs in simulated rural driving. Appl Ergon (Special Issue on Transportation Safety) 2011;42:548-54.

40 Matthews CE, Jurj AL, Shu X-o, et al. Influence of exercise, walking, cycling, and overall nonexercise physical activity on mortality in Chinese women. Am J Epidemiol 2007:165:1343-50.

41 Andersen LB, Schnohr P, Schroll M, et al. ALl-cause mortality associated with physical activity during leisure time, work, sports, and cycling to work. Arch Intern Med 2000;160:1621-8. 